\title{
The Correlation Study of C5 Nerve Root Palsy and Common Body Position in Posterior Total Laminectomy Decompression and Instrumentation
}

\author{
Tielong LIU*, Jinhai KONG*, Weiwei ZOU*, Zhengwang SUN, Wangjun YAN, Jianru XIAO \\ Second Military Medical University, Changzheng Hospital, Department of Orthopedic Oncology, Shanghai, China \\ *Tielong LIU, Jinhai KONG, Weiwei ZOU equally contributed to this study and should be considered as first authors.
}

\section{ABSTRACT}

AIM: To compare both the clinical characteristics and incidence of postoperative cervical nerve root palsy in cervical spondylosis patients treated by posterior total laminectomy, decompression and instrumentation with two different common body positions, traction bed and plaster bed.

MATERIAL and METHODS: One hundred and thirty-three patients, with a mean age of 49.5 years suffering from multilevel cervical spondylosis and treated by laminectomy from 2007 to 2009 , were reviewed.

RESULTS: Overall 30 of 133 (22.56\%) patients had C5 palsy, of which 14 (19.44\%) out of 72 patients were in the plaster bed group, while 16 of $61(26.23 \%)$ patients were in the traction bed group. The difference was statistically significant $(P<0.05)$.

CONCLUSION: The cervical curvature index $(\mathrm{CCl})$ reflected both the change of cervical height and the change of the overall cervical curvature. $\mathrm{CCl}$ was related with the incidence of $\mathrm{C} 5$ nerve root palsy postoperatively. The patients in the traction bed group had a higher nerve root palsy rate and change in cervical alignment because of over traction.

KEYWORDS: Cervical spondylosis, Laminectomy, Surgical complication, Postoperative C5 palsy, Spinal instrumentation.

\section{INTRODUCTION}

Laminectomy and posterior internal fixation is a common treatment method for cervical spondylosis. However, C5 palsy can be a significant postoperative complication (3-5, $8-10,12,13,16,17)$. Patients develop of sensation disorder and/or persistent pain around the shoulder region which is dominated by C5. Moreover, there is muscle weakness, or only motor motion weakness in the C5 sector $(15,17)$. A traction bed (Figure 1) or a plaster bed (Figure 2) are two common body positions used during this operation. These two different methods may be associated with the incidence of C5 palsy after surgery.
Our study compared the incidence and radiological and clinical characteristics of C5 palsy in those two groups. The incidence of C5 palsy combined with posterior decompression and fusion are also discussed in relation to the findings.

\section{MATERIAL and METHODS}

The data of 133 patients suffering cervical spondylosis operated by laminectomy and hardware fusion at the authors' institutions from 2007 to 2009 were reviewed. The average clinical postoperative follow-up period was 6 months (range: 3 to 12 months). One hundred and thirty three of the 133 patients who had complete sets of clinical and radiographic data were screened. 89 patients were male and 44 were female. The 
mean age at the time of surgery was 47.5 years (range: 24 to 83 years). Progressive myelopathy, 3 or more vertebral levels involved with spinal canal stenosis on radiographic images and/or previous anterior cervical fusion were indications for laminectomy. The areas of decompression were from C3 to C6 ( 17 cases), $\mathrm{C} 3$ to $\mathrm{C} 7$ ( 51 cases), $\mathrm{C} 1$ to $\mathrm{C} 7$ ( 6 cases), $\mathrm{C} 2$ to $\mathrm{C} 7$ ( 37 cases), C2 to C6 (16 cases) and C3 to T2 (6 cases). Additional decompression included laminectomy of the posterior arch at $\mathrm{C} 1$ and dome laminotomy at C2. Titanium alligation rods and lateral mass screws were used in 89 patients and pedicle screws with titanium rods were used in 44 patients.

A cervical brace was used for supporting external stabilization for 6 weeks after surgery. The concept of postoperative C5 palsy in this study was paresis of the deltoid muscle and/or sensory deficits with intractable pain in the shoulder region (C5 dermatome area) that were found after cervical spine surgery without deterioration of myelopathy. The Japanese Orthopaedic Association (JOA) scoring system (12) was used to measure the severity of myelopathy.

The clinical function and physical therapy of patients with C5 palsy were assayed according to the following: Patients in which the manual muscle test (MMT, Grade 5: no muscle weakness; Grade 0: no muscle contraction) of the deltoid muscle indicated more than one grade of muscle depraved after operation were classified as severe. Patients without muscle palsy, but who had apparent C5 dermatome area sensory deficit or pain alone were categorized as mild. The others were categorized as moderate.

\section{Radiographic Examination}

All cases had standard radiographic studies. Radiographic films were reviewed to insure patients had a coincident standing stance posture, relaxed both shoulders and level vision to see straight front. Obviating "soldier posture" and other positions that may affect stance posture, such as a lowered chin $(6,13)$. X-ray were focused at the center of the C4 level and the distance of projection was $1.5 \mathrm{~m}$, which was named standard sagittal view, and the upper boundaries of the radiograph films were parallel to the hard palate (13). We previewed the images to evaluate whether they were standardized with feasible results through preliminary radiographic scanning before taking actual projection. The measuring software can be programmed into the imaging system and uploaded into the database for individual review and measurement. Direct View software (Kodak) by 3 independent spine surgeons.

Studies were marked with serial numbers and were randomly assigned into three groups. Each study group was then randomly picked up by three surgeons whereby all study groups were rotated until each data group had been measured and reviewed by every surgeon. Neck alignment was measured by the Cobb method (Figure 3). The vertebral angle between $\mathrm{C} 2$ and $\mathrm{C} 7$ was estimated by the profile visualized in the plain radiographs in the neutral position before and after surgery. From this, the change rate of curvature was determined. For the cervical curvature index (CCl) (Figure 4), the preoperative and postoperative cervical alignments were evaluated from the lateral radiographs of the cervical spine in the neutral position. The JOA score was used to evaluate the severity of and improvement in cervical myelopathy. The total JOA score for a healthy patient is 17 points. The recovery rate was calculated from the surgical scores assessed preoperatively, postoperatively and at the 6-month follow up.

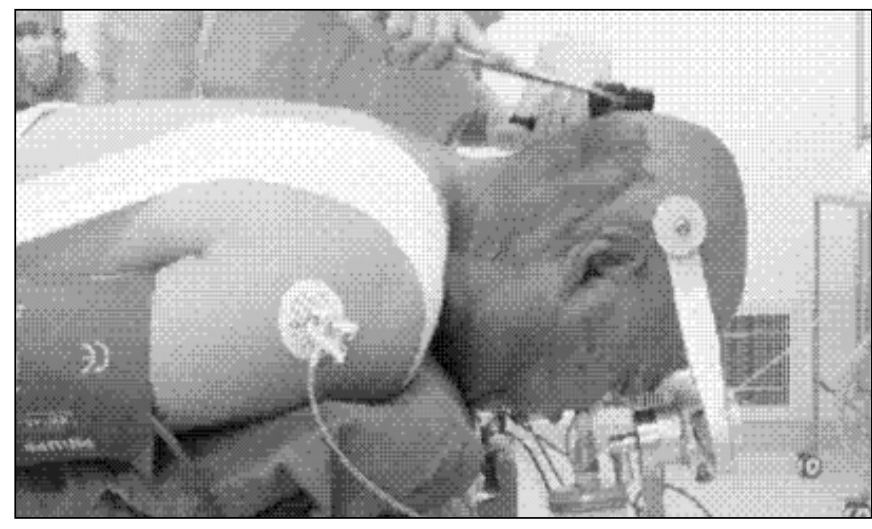

Figure 1: The patient during the operation in the traction bed.

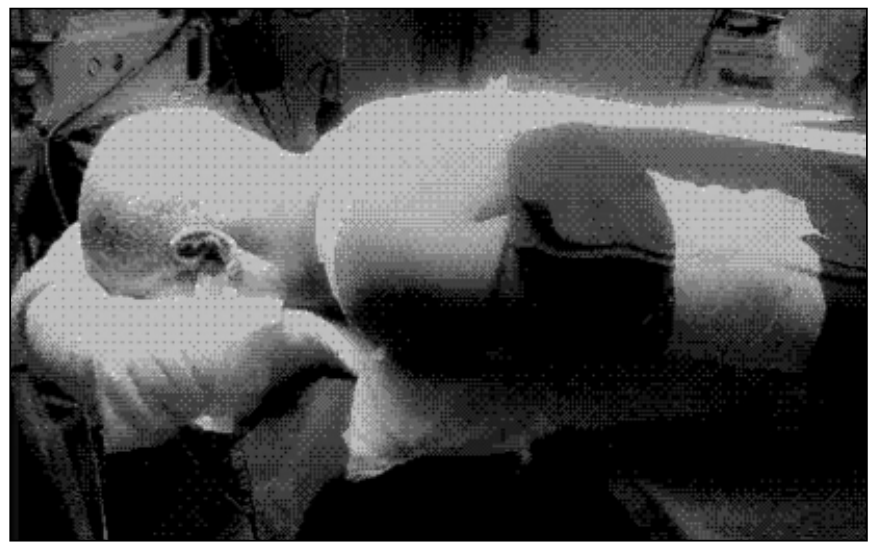

Figure 2: The patient during the operation in the plaster bed.

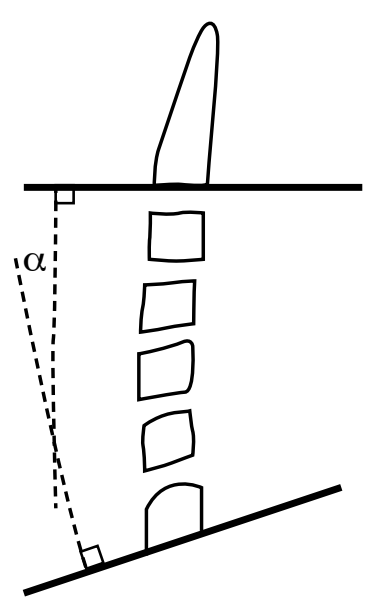

Figure 3: The Cobb angle is defined as the angle formed between a line drawn parallel to the inferior endplate of $\mathrm{C} 2$ and a line drawn parallel to the inferior endplate of $\mathrm{C} 7$. 


\section{Statistical Analysis}

The incidence of postoperative C5 palsy and neurological function recovery rate (JOA score) in patients from the plaster group was compared with those from the traction group using univariate analysis. The unpaired t-test was used to compare the preoperative and postoperative mean curvature between the groups, the mean $\mathrm{CCl}$ index between the groups, and the change rate of curvature and change rate of $\mathrm{CCl}$ index comparison between the groups. Statistical significance was $p<0.05$. The mean standard deviation was reported for all values.

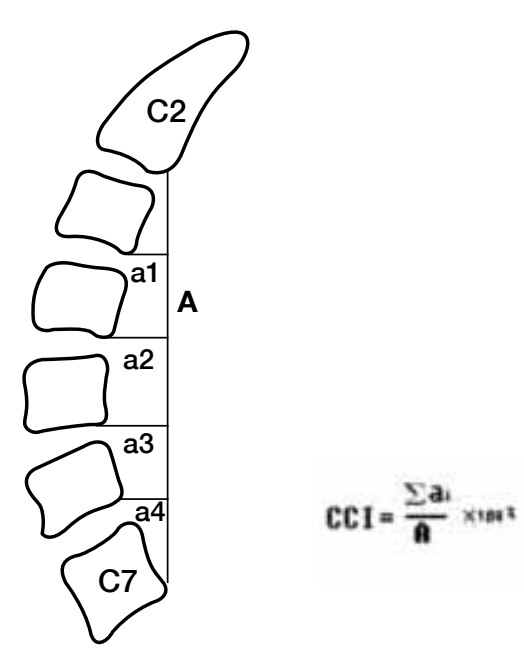

Figure 4: Cervical curvature index (Ishihara). Line segment $A$ is drawn between the posteroinferior edge of the $\mathrm{C} 2$ and $\mathrm{C} 7$ vertebral bodies. The four lines, a1-a4, are drawn perpendicular to line A from each posteroinferior edge of the C3,C4, C5, and C6 vertebral bodies. The cervical curvature index is the percentage of the sum of the four segments divided by line segment $A$.

\section{RESULTS}

Overall, C5 palsy was found in 30 of 133 (22.56\%) patients, of which $14(19.44 \%)$ of 72 cases were in the plaster bed group, while 16 of $61(26.23 \%)$ cases were in the traction bed group (Table I). This difference was statistically significant $(p<0.01)$, which is risky regarding developing C5 palsy. The risk when using the traction bed was 1.35 times higher versus using the plaster bed. The mean preoperative and postoperative cervical curvatures between the traction and plaster groups are shown in Table II. The mean preoperative cervical curvature was $10.27 \pm 5.98$ and $9.92 \pm 5.77$, respectively. The difference was not statistically significant ( $p>0.05$ ).

The mean postoperative cervical curvature was $11.93 \pm 5.02$ and $13.51 \pm 6.19$, respectively, and the difference was also not statistically significant $(p>0.05)$. However, there were statistically significant differences between the preoperative cervical curvatures and the postoperative cervical curvatures in the plaster group $(p<0.05)$. The mean preoperative and postoperative $\mathrm{CCl}$ index of both groups are shown in Table III. The only statistical difference was between the preoperative values in the plaster group. The preoperative $\mathrm{CCl}$ index and the postoperative $\mathrm{CCl}$ index in the traction group were $8.32 \pm 3.77$ and $8.67 \pm 5.23$, respectively. The comparison between the change rate of cervical curvature and change rate of $\mathrm{CCl}$ index is shown in Table IV. The pre- and postoperative change rate of cervical curvature in the plaster group and the traction groups was $16.17 \pm 6.97$ and $36.19 \pm 12.53$, respectively, with the difference being statistically significant $(p<0.05)$. The pre- and postoperative $\mathrm{CCl}$ index change rate in the plaster group and the traction group was $4.21 \pm 1.92$ and $6.84 \pm 2.17$, respectively, which was also significantly different $(p<0.05)$. The mean preoperative JOA score, postoperative JOA score and the improvement rate are shown in Table V. The neurological function recovery rate in the two groups was not significantly different $(p>0.05)$.

Table I: The Nerve Root Palsy Degree of the Traction Group and the Plaster Group

\begin{tabular}{c|c|ccc|c}
\hline & \multirow{2}{*}{$\begin{array}{c}\text { Number of } \\
\text { Patients }\end{array}$} & severe & moderate & Nerve root palsy & Percentage \\
\cline { 3 - 6 } & 61 & 7 & 6 & 3 & $26 \%$ \\
\hline Traction & 72 & 4 & 5 & 5 & $19 \%$ \\
\hline Plaster & 133 & 11 & 11 & 7 & $22 \%$ \\
\hline Total & & &
\end{tabular}

Table II: The Preoperative and Postoperative Mean Curvature in the Two Groups

\begin{tabular}{lccc}
\hline & A & B & p value \\
\hline Number of Patients & 61 & 72 & $>0.05$ \\
\hline Preoperative Mean Curvature & $10.27 \pm 5.98$ & $9.92 \pm 5.77$ & $>0.05$ \\
\hline Postoperative Mean Curvature & $11.93 \pm 5.02$ & $13.51 \pm 6.19$ & $<0.05$ \\
\hline p value & $>0.05$ & & \\
\hline
\end{tabular}


Table III: The Preoperative and Postoperative Mean CCI in the Two Groups

\begin{tabular}{lccc}
\hline & A & B & p value \\
\hline Number of patients & 61 & 72 & $>0.05$ \\
\hline Preoperative Mean CCl & $8.32 \pm 3.77$ & $8.19 \pm 3.92$ & $>0.05$ \\
\hline Postoperative Mean CCI & $8.67 \pm 5.23$ & $8.75 \pm 4.96$ & $<0.05$ \\
\hline p value & $>0.05$ & & \\
\hline
\end{tabular}

CCI: Cervical curvature index (Ishihara)

Table IV: The Change Rate of the Curvature and $\mathrm{CCl}$ in the Two Groups

\begin{tabular}{lccc}
\hline & A & B & p value \\
\hline Number of patients & 61 & 72 & $>0.05$ \\
\hline Change rate of curvature & $16.17 \pm 6.97$ & $36.19 \pm 12.53$ & $>0.05$ \\
\hline Change rate of $\mathrm{CCl}$ & $4.21 \pm 1.92$ & $6.84 \pm 2.17$ & \\
\hline
\end{tabular}

Table V: The Preoperative and Postoperative JOA Score in the Two Groups

\begin{tabular}{lccc}
\hline & Preoperative JOA Score & Postoperative JOA Score & Improvement Rate \\
\hline A & $9.52 \pm 3.15$ & $14.42 \pm 2.72$ & $63.21 \pm 35.32 \%$ \\
\hline B & $9.49 \pm 3.29$ & $14.51 \pm 2.93$ & $69.32 \pm 38.57 \%$ \\
\hline
\end{tabular}

\section{DISCUSSION}

This study sought to determine the incidence of C5 palsy, a common postoperative complication in patients that have undergone a decompressive laminectomy using two common body positions, traction bed and plaster bed. Out of 133 patients reviewed who underwent a posterior decompressive laminectomy secondary to myelopathy, $22 \%$ of them presented with C5 nerve root palsy. Radiographic data from these patients were independently reviewed to determine the predisposing factors that may result in this complication. The mean curvature, change rate of curvature, $\mathrm{CCl}$ index and change rate of $\mathrm{CCl}$ index were compared. The results of our study suggest that the incident rate of C5 palsy is significantly higher in patients in the traction bed position as compared to the plaster bed position. There were statistically significant changes in the mean $\mathrm{CCl}$ index and change rate of curvature postoperatively as compared to preoperatively in the traction group, with no statistical change in the plaster group. In addition, the change rate of curvature and change rate of $\mathrm{CCl}$ index were significantly greater in the traction group as compared to the plaster group. These results suggest that excessive traction can occur while using the traction bed body position leading to C5 palsy in these patients.

In a previous review of patients treated with posterior decompressive laminectomy, the incidence rate was $4.6 \%$ (range:0\% to $30 \%$ ) (7). The results of our study show a higher incidence rate of $22 \%$. It is not clear to us why our incidence rate is higher. However, there are certain clinical factors that may affect the incidence rate. There are differences in surgical techniques $(2,12,16)$, and/or inclusion criteria of the study (13). In addition, the concept of C5 palsy needs to be defined for different institutes to compare the studies. Postoperative paresis of the upper extremities can develop after cervical surgery and the most common nerve is C5. Most C5 palsies have been shown to occur unilaterally and have little effect on the muscles bilaterally $(6,10,18)$.

The most common C5 palsies were detected around a week after the operation $(4,20)$. The latency period may be longer, from 2 to 4 weeks in some cases (4). Other cervical nerve palsies $(\mathrm{C} 6, \mathrm{C} 7$, or $\mathrm{C} 8)$, in isolation or combination, have been reported with lower incidence $(4,15)$. Unfortunately, the pathomechanism of C5 palsy after laminectomy is unknown, although there are several hypotheses regarding its etiology $(4,13)$ :

(1) C5 palsy is caused by tightening up of the nerve root when shifting posteriorly to the spinal cord and it is also relevant to anchoring the nerve root at the edge of the uncovertebral joint and/or the superior facet $(5,16,17,19)$.

(2) latrogenic nerve root injury during operation (11).

(3) Spinal cord reperfusion injury during operation (4).

(4) Reperfusion injury leads to radicular artery blood supply decrease and causes ischemia. Consequently, for patients with posterior laminectomy and instrumented fusion, other risk possibilities may also be present (4). For example, instrumentation damage, "lag correction effect", 


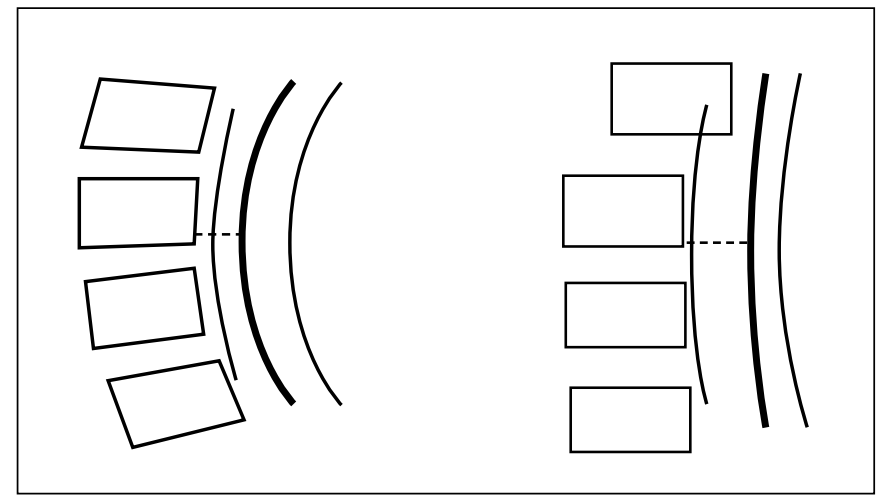

Figure 5: When the height of the cervical vertebra after traction is increased and the cervical curvature decreased, the front margin of the vertebra was relatively pexic, while the posterior gap increased and the overall height of the posterior margin of the centrum increased.

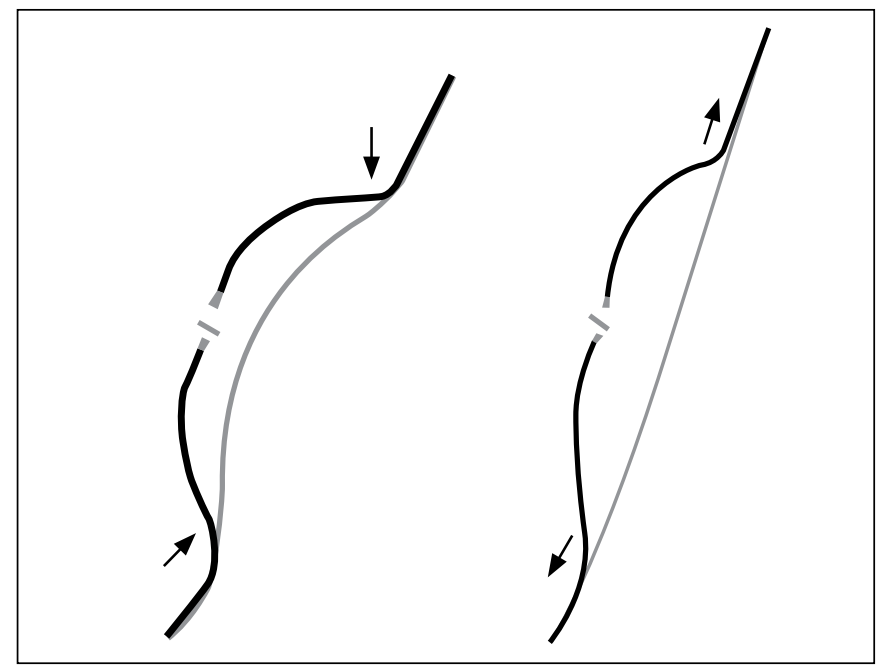

Figure 6: When a bow is forcibly bent outward, the bow naturally spreads resulting in the bowstring (spinal cord) being gradually tensed. Though the bow depth decreased, the distance between the bowstring and bow-top obviously increased and traction between the nerve root and spinal cord appeared.

pulling the anterolisthetic vertebra posteriorly may cause iatrogenic foraminal stenosis $(1,13)$. However, all of these are assumptions regarding C5 palsy development and do not have significant evidence supporting them. Exact proof with sufficient research samples, scientific analysis and clear radiographic films demonstrating the injured nerve root injured has not been reported (7). C5 nerve palsy can be detected by the weakness of the deltoid muscle and the degree may also indicate the prognosis of the patient. Most of the cases were fully recovered within six months in this study, while those cases with a deltoid strength higher than MMT grade 3 after surgery recovered faster than cases with only MMT grade 1. All the patients of the mild group recovered to MMT grade 5 within 3 months. The other published paper indicated similar findings $(1,10)$. In the current study, the average change rate of $\mathrm{CCl}$ index for the plaster bed group was more than $2.63 \%$ compared with the traction bed group $(\mathrm{p}<0.05)$. The $\mathrm{CCl}$ index reflected both the change of cervical height and the change of the overall cervical curvature, so excessive intraoperative traction and the use of internal fixation may have resulted in the increase of C5 nerve root palsy.

When the height of cervical vertebra after traction increased and the physiological curvature decreased, the front margin of the vertebra was relatively pexic, while the posterior gap increased and the overall height of the posterior margin of the centrum increased. The spinal cord within the spinal canal was gradually distracted and drifted backward (Figure 5). This would be analogous to a forcibly bent bow naturally spread (Figure 6). With the bow spreading, the bowstring (spinal cord) was gradually tensed. Though the bow depth decreased, the distance between bowstring and bow-top obviously increased and traction occurred between the nerve root and spinal cord.

The C5 nerve root was on the top of the cervical spinal cord and would obviously be stretched; at this time palsy would be the most common. It seemed that this theory could also explain why it was easier for the signal change of the adjacent spinal segment for patients with nerve root palsy to emerge. In addition, traction of the spinal cord would cause internal vasospasm leading to vasoconstriction. If elongated in this way, it would cause a local metabolic disorder, and spinal cord signal would change or worsen. The solution of reducing the incidence of C5 palsy after operation could include decreasing the tension on the C5 nerve root, for instance, a prophylactic foraminotomy (12), durotomy (16) or limiting shifting of the posterior section of the spinal cord by preventing aggressive opening of the lamina during laminectomy $(13,14)$.

Additional cervical alignment must be considered. Takemitsu et al believe that the roots and spinal cord tension can be increased when correcting the alignment from the posterior of the cervical spine using instrumentation more than without correction (13). Our study also found that the patients in the traction bed group have much higher nerve root palsy rate and a significant change in cervical alignment because of excessive traction. Therefore, we believe that avoiding excessive traction during surgical decompression procedures and appropriate traction relaxation before final fastening may reduce the incidence of C5 nerve root palsy. Patients undergoing decompressive laminectomy using a traction bed for patient positioning have a much higher C5 palsy rate than those patients who were treated using a plaster bed for patient positioning. There was a statistically higher change in cervical alignment, likely caused by overtraction. The $\mathrm{CCl}$ index reflected both the change of cervical height and the change of the overall cervical curvature, and is related to postoperative C5 nerve root palsy. Avoiding excessive intraoperative traction and providing appropriate traction relaxation during decompression procedures before final fastening may reduce the incidence of $\mathrm{C} 5$ nerve root palsy.

\section{CONCLUSION}

The incidence rate of cervical spondylosis patients who 
developed cervical nerve root palsy after treatment by posterior total laminectomy decompression and instrumentation using two different common body positions, traction bed and plaster bed was determined. A statistically higher incidence of postoperative C5 palsy association with cervical alignment was found in patients where the traction bed was used. Excessive traction should be avoided when performing instrumented decompressive laminectomy.

\section{REFERENCES}

1. Abumi K, Shono $\mathrm{Y}$, Ito $\mathrm{M}$, Taneichi H, Kotani $\mathrm{Y}$, Kaneda K: Complications of pedicle screw fixation in reconstructive surgery of the cervical spine. Spine 25: 962-969, 2000

2. Boakye M, Patil CG, Ho C, Lad SP: Cervical corpectomy: Complications and outcomes. Neurosurgery 63: 295-301, 2008

3. Chen Y, Chen D, Wang X, Guo Y, He Z: C5 palsy after laminectomy and posterior cervical fixation for ossification of posterior longitudinal ligament. J Spinal Disord Tech 20(7):533-535, 2007

4. Chiba K, Toyama $\mathrm{Y}$, Matsumoto $\mathrm{M}$, Maruiwa $\mathrm{H}$, Watanabe $\mathrm{M}$, Hirabayashi K: Segmental motor paralysis after expansive opendoor laminectomy. Spine 27:2108-2115, 2002

5. Epstein JA: Extradural tethering effects as one mechanism of radiculopathy complicating posterior decompression of the cervical spinal cord. Spine 21(15):1839-1840, 1996

6. Hasegawa K, Homma T, Chiba Y: Upper extremity palsy following cervical decompression surgery results from a transient spinal cord lesion. Spine 32(6): E197- 202, 2007

7. Kalisvaart MM, Nassr A, Eck JC: C5 palsy after cervical decompression procedures. Neurosurg Q 19(4):276-282, 2009

8. Komagata M, Nishiyama M, Endo K, Ikegami H, Tanaka S, Imakiire A: Prophylaxis of C5 palsy after cervical expansive laminectomy by bilateral partial foraminotomy. Spine J 4(6):650-655, 2004

9. Sakaura H, Hosono N, Mukai Y, Fujii R, Iwasaki M, Yoshikawa $\mathrm{H}$ : Segmental motor paralysis after cervical laminoplasty: A prospective study. Spine 31(23): 2684-2688, 2006
10. Sakaura H, Hosono N, Mukai $Y$, Ishii T, Yoshikawa H: C5 palsy after decompression surgery for cervical myelopathy: Review of the literature. Spine 28(21):2447-2451, 2003

11. Satomi K, Nishu Y, Kohno T, Hirabayashi K: Long-term follow-up studies of open- door expansive laminectomy for cervical stenotic myelopathy. Spine 19(5): 507-510, 1994

12. Sasai K, Saito T, Akagi S, Kato I, Ohnari H, lida H: Preventing C5 palsy after laminectomy. Spine 28: 1972-1977, 2003

13. Takemitsu M,Cheung KM, Wong YW, Cheung WY, Luk KD: C5 nerve root palsy after cervical laminoplasty and posterior fusion with instrumentation. J Spinal Disord Tech 21(4): 267-272, 2008

14. Tokuhashi Y, Matsuzaki H, Wakabayashi K: Postoperative cervical radiculopathy after laminoplasty. Bessatsu Seikeigeka 29:195199, 1996 (in Japanese)

15. Tsuzuki N, Abe R, Saiki K, Okai K: Paralysis of the arm after posterior decompression of the cervical spinal cord. II; Analyses of clinical findings. Eur Spine J 2(4):197-202, 1993

16. Tsuzuki N, Abe R, Saiki K, Zhongshi L: Extradural tethering effect as one mechanism of radiculopathy complicating posterior decompression of the cervical spinal cord. Spine 21:203-211, 1996

17. Uematsu Y, Tokuhashi Y, Matsuzaki H: Radiculopathy after laminectomy of the cervical spine. Spine 23: 2057-2062, 1998

18. Wada E, Suzuki S, Kanazawa A, Matsuoka T, Miyamoto S, Yonenobu K: Subtotal corpectomy versus laminoplasty for multilevel cervical spondylotic myelopathy: A long- term- followup study over 10 years. Spine 26(13):1443-1447, 2001

19. Xu-Hui Z, Jia-Hu F, Lian-Shun J, Zhi-Yong C, Yong Z, XiongSheng C, Wei-Ping W: Clinical significance of cervical vertebral flexion and extension spatial alignment changes. Spine (Phila Pa 1976) 34(1):E21-26, 2009

20. Yamashita T, Yokogusu K, Yokozawa H: C5 nerve palsy after cervical laminoplasty: An analysis of three cases. Seikei Geka 47: 1365-1369, 1996 